\title{
Optimization of Multi-size Micro-perforated Panel Absorbers with Different Cavity Depths using Adaptive Genetic Algorithm
}

\author{
Zhenglian $\mathrm{Lu}^{+}$ \\ IOT Engineering College, HoHai University, Chang Zhou, China
}

\begin{abstract}
With the development of society, noise pollution has become more and more serious. How prevent and control the noise pollution to get the proper acoustic environment has been the hotspot. Therefore, a broad band sound absorber which is thin, durable, clean and environmentally friendly is highly demanded in modern society. In this paper, a new type of multiple size micro-perforated panel (MPP) absorbers with different cavity depths is proposed and studied. Firstly, a mathematical model for multi-size MPP absorbers with different cavity depths is developed. Thereafter, based on the established model, adaptive genetic algorithm (AGA) is proposed in this study as a tool to solve the optimization problem of finding the best combination of the constitutive parameters of multi-size MPP absorbers with different cavity depths providing the maximum average absorption within a prescribed frequency range. Results show that AGA provides a fast and effective method to solve the structure optimization of multi-size MPP absorbers with different cavity depths.
\end{abstract}

Keywords: multi-size MPP absorber, different cavity depths, adaptive genetic algorithm (AGA), optimization

\section{Introduction}

It is well known that noise can seriously hurt people's physical and mental health. Based on the investigations carried out in 1970 by the Occupational Safety and Health Act (OSHA), noise is largely responsible for the psychological and physical illness of workers. Therefore noise control has become increasingly important and pressing as technology develops and society evolves. An MPP absorber with uniform-size holes [1-3] has been regarded as a promising clean alternative to the traditional porous soundabsorbing materials which are environmentally-unfriendly. Since Maa's pioneering works [1], the MPP absorber is always one research focus and has been used in various fields [4-7]. However, a uniform-size MPP absorber is usually insufficient for a general purpose absorber because it is effective only in a narrow band around its resonance frequencies. And thus, in efforts to widen the absorption bandwidth, numerous studies have been performed [1, 2, 8, 9]. Introducing more resonance frequencies is an effective way to widen the absorption bandwidth, such as multi-layer MPP absorbers, however, which will result in thicker sound absorbers because the panels are arranged in series [10]. And many researchers keep studying and finding more new and better ways to introduce more resonance frequencies without significant increase in thickness of the absorbing structure. One is to use holes of multiple sizes for MPP absorbers instead of a uniform holes size $[11,12]$. Another is to use partitioned cavity of varying depths for MPP absorbers instead of a constant cavity depth [13]. With these type of MPP absorbers that have perforations of multiple sizes or cavities of different depths, a wider sound absorption bandwidth and thinner sound absorber is achievable, and which would be very beneficial when only a limited space is available to install the sound absorber. So far, however, few studies have combined the advantages of multi-size holes and different depths of cavities. One possible reason is that such structure is very complicated which greatly increases the difficulty and

\footnotetext{
+ Corresponding author. Tel.: +86 13601501578

E-mail address: 19931458@hhu.edu.cn
} 
complexity of structure design. Since the absorption performance of a uniform-size MPP absorber with a constant cavity depth depends on only four parameters - the hole diameter, the perforation ratio, the panel thickness and the cavity depth, while the design of an $N$-size MPP absorber with respective different depths of cavities involves the tuning of $3 N+1$ parameters usually greater than those of a uniform-size MPP absorber with a constant depth of cavity, and which makes it difficult to design in a practical application.

From an engineering point of view, here the interest is to obtain the combination of structure parameters within given variation ranges that provides the maximum mean absorption for a specified frequency range. This is typically an optimization problem. In this paper, as an improved genetic algorithm, adaptive genetic algorithm (AGA) is proposed and used as a new attempt to optimize the mean absorption of a multi-size MPP absorber with different cavity depths within given variation ranges of constitutive parameters.

\section{Incidence Sound Absorption Of Various MPP Absorbing Construction}

\subsection{Two-size MPP absorber with different cavity depths}

Unlike the traditional uniform-size MPP absorber [1], a two-size MPP absorber proposed in this paper has partitioned cavities of different depths and two sizes of holes that are of parallel arrangement, as shown in Fig. 1. Only in this way [16], the benefit from each size of holes or cavities can be combined.

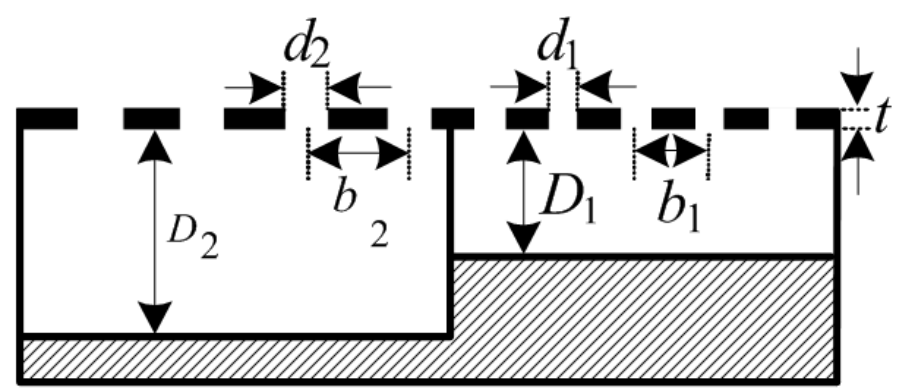

Fig. 1: Schematic diagrams of two-size MPP absorbers with two partitioned cavities

In the present theoretical model, the panel is assumed to be rigid that will not vibrate under acoustic loading. The analysis is based on the flow continuity of air on both sides of MPP. As shown in Fig. 2, $\bar{v}_{1}$ and $\bar{v}_{2}$ is the mean particle velocity of surrounding air under any acoustic loading with respectively pressure difference $\Delta p_{1}$ and $\Delta p_{2}$ are respectively for two-size holes.

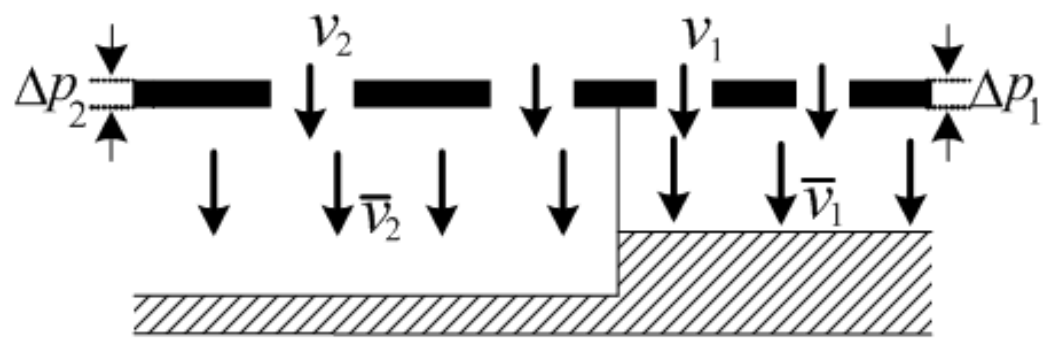

Fig. 2: Analytical model of a multi-size MPP with diferent cavity depths

The continuity of the volume velocity gives the following formulation for the average particle velocity of the surrounding air in the vicinity of both sides of the MPP:

$$
\bar{v}_{1}=v_{1} \sigma_{1}, \bar{v}_{2}=v_{2} \sigma_{2}
$$

Where $v_{1}$ and $v_{2}$ are the spatially averaged velocity in the holes of diameter $d_{1}$ (namely hole 1 in the following expression) and in the holes of diameter $d_{2}$ (namely hole 2 in the following expression), respectively, and $\sigma_{1}$ and $\sigma_{2}$ are respectively the perforation ratio of hole 1 and hole 2. Let $z_{1}$ and $z_{2}$ be the specific acoustic impedance of the hole 1 and hole 2 , respectively, which are defined as

$$
z_{1}=\Delta p_{1} / v_{1}, z_{2}=\Delta p_{2} / v_{2}
$$

The specific acoustic impedance of the air cavities are given by

$$
z_{c 1}=-j \rho c \cot \left(\omega D_{1} / c\right) \quad, z_{c 2}=-j \rho c \cot \left(\omega D_{2} / c\right)
$$


Since the partition panel is perpendicularly installed between the MPP and the back wall (see Fig. 2) which will provide separate cavity for each size holes. In such case, the total specific acoustic impedance $z_{\text {total }}$ can be given by

$$
z_{\text {total }}=\left(1 /\left(\frac{\Delta p}{\sigma_{1} v_{1}}+z_{c 1}\right)+1 /\left(\frac{\Delta p}{\sigma_{2} v_{2}}+z_{c 2}\right)\right)^{-1}=\left(\frac{1}{z_{1} / \sigma_{1}+z_{c 1}}+\frac{1}{z_{2} / \sigma_{2}+z_{c 2}}\right)^{-1}
$$

According to the equivalent circuits, their total relative specific acoustic impedance can be calculated as

$$
z_{\text {total }}=\left(\frac{1}{r_{1}+j \omega m_{1}-j \cot \left(\omega D_{1} / c\right)}+\frac{1}{r_{2}+j \omega m_{2}-j \cot \left(\omega D_{2} / c\right)}\right)^{-1}
$$

Where $r_{1}+j \omega m_{1}$ in equation (18) denotes the relative specific acoustic impedance of hole $1, r_{2}+j \omega m_{2}$ is the relative specific acoustic impedance of hole 2 , and $-j \cot \left(\omega D_{1} / c\right)$ and $-j \cot \left(\omega D_{1} / c\right)$ is the relative specific acoustic impedance of their back cavity As can be seen from equation (18d), two resonant absorption peaks may be introduced provided that the structure parameters are appropriately designed.

\subsection{Genetic Algorithm}

Genetic algorithm (GA) is an adaptive search technique and has been applied to a variety of problems [16]. GA is based on mechanics of natural genetics and natural selection. The artificial survival of the fittest is combined with genetic operators which are abstracted from nature. As an improvement for simple genetic algorithm (SGA), adaptive genetic algorithm (AGA) develops the adaptive crossover probability and mutation probability in the process of evolution which can not only make that the group search towards a good direction more quickly, but also make it combat premature convergence inherent in SGA. So AGA will work well for our problem domain where the global optimal value is searched for by given a fitness function. In the following, we give a short description of the AGA, which is applied as the optimizer in the sound absorption performance optimization of multi-size MPP absorbers with partitioned cavities of different depths.In this study, the objective function is $\langle a\rangle=\sum a\left(f_{i}\right) /\left(f_{u}-f_{l}\right)$, where $f_{l}$ and $f_{u}$ represent the lower

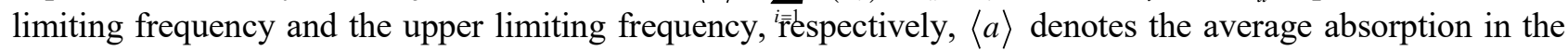
frequency band $\left(f_{l}, f_{u}\right)$ and $a\left(f_{i}\right)$ denotes the absorption coefficient corresponding to the frequency $f_{i}$. The step size between $f_{l}$ and $f_{u}$ is 1 . Under this condition, $N_{0}$ equals to $f_{u}-f_{l}$ and $f_{i+1}$ is equivalent to $f_{i}+1$. The block diagram of the AGA procedure is depicted in Fig. 3.

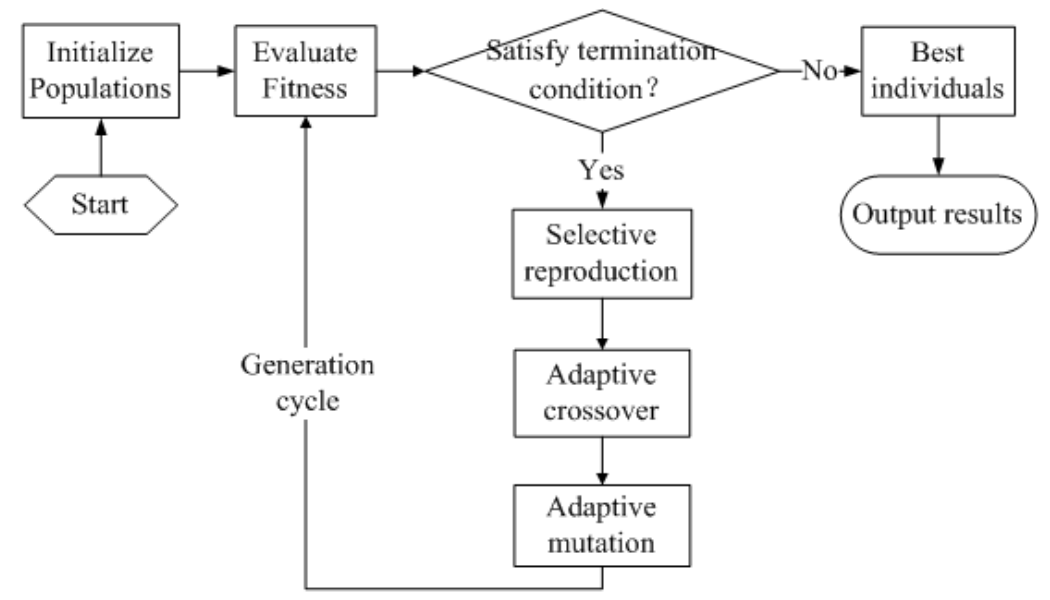

Fig. 3: Block diagram of MPGA procedure

\subsection{Case Study}

Please acknowledge collaborators or anyone who has helped with the paper at the end of the text. The optimization system is programmed by MATLAB and run in Lenovo PC-Intel. In our program, the panel thickness is kept fixed according to the actual demand, and therefore the decision variables will be reduced which makes the algorithm more efficient and easy to run. Consequently, for a two-size MPP absorber with different cavity depths, there are six decision variables, the hole diameters $d_{1}, d_{2}$, the perforation ratio $\sigma_{1}, \sigma_{2}$ and the cavity depths $D_{1}$ and $D_{2}$ Similarly, there are nine decision variables for a three-size MPP absorber, the hole diameters $d_{1}, d_{2}, d_{3}$, the perforation ratio $\sigma_{1}, \sigma_{2}, \sigma_{3}$ and the cavity depth $D_{1}, D_{2}, D_{2}$. 
Suppose a two-size MPP absorber with different cavity depths is designed to reduce noise in the frequency range of $100 \sim 4000 \mathrm{~Hz}$. The panel thickness $t$ is kept fixed at $0.5 \mathrm{~mm}$ based on the actual demand and cost constraints. Based on the current processing technology and practical application, a series of assumptions of the constrained condition in design are illustrated as

$$
0.1 \mathrm{~mm} \leq d_{1}, d_{2} \leq 1 \mathrm{~mm}, 0.1 \% \leq \sigma_{1}, \sigma_{2} \leq 16 \%, 0.005 \mathrm{~m} \leq D_{1}, D_{2} \leq 0.1 \mathrm{~m}
$$

SGA is applied to optimize this two-size MPP absorber within given variation ranges. The procedure comes to an end at the 150th generation. As stated above, the objective function to be maximized is the average absorption in the frequency band $\left(f_{l}, f_{u}\right)$ with $f_{l}=100 \mathrm{~Hz}$ and $f_{u}=4000 \mathrm{~Hz}$. To evaluate the performance of AGA, we compare it to SGA that has the same population size. Table 1 shows structure parameters (the panel thickness is kept constant at $0.5 \mathrm{~mm}$ ).

Table 1: Optimized Structure Parameters of Two-size MPP Absorbers

\begin{tabular}{|c|c|l|l|l|l|l|}
\hline & \multicolumn{7}{|c|}{ Structure Parameters } \\
\cline { 2 - 7 } & $\boldsymbol{d}_{\mathbf{1}}(\mathbf{m m})$ & \multicolumn{1}{|c|}{$\boldsymbol{d}_{\mathbf{2}}(\mathbf{m m})$} & $\sigma_{\mathbf{1}}(\boldsymbol{\%})$ & $\boldsymbol{\sigma}_{\mathbf{2}}(\boldsymbol{\%})$ & $\boldsymbol{D}_{\mathbf{1}}(\mathbf{m})$ & \multicolumn{1}{c|}{$\boldsymbol{D}_{\mathbf{2}}(\mathbf{m})$} \\
\hline \multirow{2}{*}{$\mathrm{AGA}$} & 0.079 & 0.132 & 8.597 & 2.672 & 0.024 & 0.1 \\
\hline SGA & 0.131 & 0.163 & 2.962 & 2.086 & 0.017 & 0.098 \\
\hline
\end{tabular}

The normal sound absorption coefficient curve relative to frequency is plotted in Fig. 5 for comparison. Fig. 4 shows that the AGA outperforms the SGA. Particularly, the absorption coefficient around the frequency of $3500 \mathrm{~Hz}$ is improved greatly.

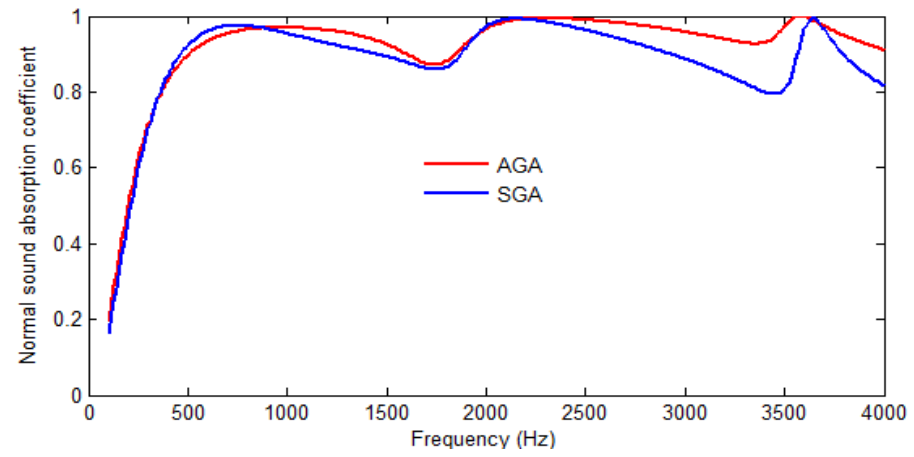

Fig.4: Optimal sound absorption coefficient for a two-size MPP absorber with different cavity depths based on AGA and SGA

\section{Conclusions}

In this paper, to fill the research gap, a multi-size micro-perforated panel (MPP) absorber with different cavity depths is presented and studied from the dual-mathematical model and optimization method. The theoretical analysis is based on the continuity of air flow on both sides of MPP. The mathematical model of normal incidence sound absorption for multi-size micro-perforated panel (MPP) absorber with different cavity depths provides a basis for the optimization method based on adaptive genetic algorithm (AGA) that has global searching ability. AGA is used to optimize the structure parameters of fitness function in order to obtain optimal sound absorption performance under given conditions. Its performance is evaluated by comparing with simple genetic algorithm (SGA) results and the available results in the literature. Results show that AGA works better at optimizing in respect of obtaining broad absorption bandwidth. This study provides the reference for using AGA when determining the best combination of the structure parameters of MPP absorbers with different cavity depths under normal incidence condition. The optimization of MPP absorbers with different cavity depths under oblique incidence condition should be expected in the near future.

\section{Acknowledgements}

This work was supported by National Natural Science Foundation of China (No. 11604077 and No. 61601169) and Jiangsu Provincial Natural Science Foundation for Youths (No. BK20150248). 


\section{References}

[1] D.Y. Maa. Theory and design of microperforated panel sound-absorbing constructions. Scientia Sinica. 1975, 18(1): 55-71.

[2] D.Y. Maa. Microperforated-panel wideband absorber. Noise control Eng. J. 1987, 29: 77-84.

[3] D.Y. Maa. Potential of microperforated panel absorbe. Ch. J. Acoust. Soc. Am. 1998, 104: 2861-2866.

[4] H.V. Fuchs and X. Zha. Creating low-noise environments in communication rooms. Appl. Acoust. 2001, 62: 1375-1396.

[5] X. Zha and H.V. Fuchs, "Improving the acoustic working conditions for musicians in small spaces", Appl. Acoust., vol. 63, pp. 203-331, 2002.

[6] M.Q. Wu. Micro-perforated panels for duct silencing. Noise Control Eng. J. 1997, 45: 69-77.

[7] J. Kang and M.W. Brocklesby. Feasibility of applying microperforated absorbers in acoustic window systems. Appl. Acoust. 2005,66: 669-689.

[8] Z.M. Zhang, X.T. Gu. The theoretical and application study on a double layer microperforated sound absorption structure. J. Sound Vibr.1998, 215:399-405.

[9] K. Sakagami, Y. Nagayama, M. Morimoto. Double-leaf microperforated panel space absorbers: A revised theory and detailed analysis. Appl. Acoust. 2009, 70:703-709.

[10] W.H. Chen, F.C. Lee and D.M. Chang. On the acoustic absorption of porous materials with different surface shape and perforated plates. J. Sound Vibr. 1996, 273:337-355.

[11] I.M. Misasa and Okuma M. An experimental study of a multi-size micro-perforated panel absorber. J. System Design Dynamics. 2007, 1(2): 331-339.

[12] D.Y. Maa. Combination of microperforated panel absorber. J. Noise Vibr. Control. 1990, 10: 3-9.

[13] C.Q. Wang and L.X. Huang. On the acoustic properties of parallel arrangement of multiplemicro-perforated panel absorbers with different cavity depths. J. Acoust. Soc. Am. 2011, 130:208-218.

[14] Y.C. Chang and L.J. Yeh. Optimization of double-layer absorbers on constrained sound absorption systerm by using genetic algorithm. Int. J. Numer. Meth. Engnn.2004, 95: 317-333.

[15] X.D. Zhao and X.J. Zhang. Three layer microperforated panel optimal design and analysis of its characteristic. Acta Acustica, 2008, 33:84-87.

[16] D.E. Goldoerg. Genetic algorithms in search, optimization and machine learning, Addison-Wesley publishing company. Boston, 1989 